\title{
Magnetoresistance Study of $c$-Axis Oriented YBCO Thin Film
}

\author{
M. Chrobak ${ }^{a}$, W.M. Woch ${ }^{a, *}$, M. Kowalik $^{a}$, R. Zalecki ${ }^{a}$, M. Giebultowski $^{a}$, J. \\ PRZEWOŹNIK $^{a}$, Cz. KAPUSTA ${ }^{a}$ AND G. SzWACHTA ${ }^{b, c}$ \\ ${ }^{a}$ Solid State Physics Department, Faculty of Physics and Applied Computer Science, \\ AGH University of Science and Technology, al. A. Mickiewicza 30, 30-059 Krakow, Poland \\ ${ }^{b}$ Department of Surface Engineering and Materials Characterization, Faculty of Metals Engineering and Industrial \\ Computer Science, AGH University of Science and Technology, al. A. Mickiewicza 30, 30-059 Krakow, Poland \\ ${ }^{c}$ Academic Centre for Materials and Nanotechnology, AGH University of Science and Technology, \\ al. A. Mickiewicza 30, 30-059 Krakow, Poland
}

\begin{abstract}
The $c$-axis orientation $\mathrm{YBa}_{2} \mathrm{Cu}_{3} \mathrm{O}_{\delta}$ thin film was prepared directly on $\mathrm{MgO}$ substrate by the pulse laser deposition. The thickness of the film is $170 \mathrm{~nm}$. The superconducting critical temperature is $T_{c 50 \%}=87.5 \mathrm{~K}$ and the width of superconducting transition is $\Delta T=1.8 \mathrm{~K}$. The temperature dependences of magnetoresistance were measured up to $90 \mathrm{kOe}$. The widths of the transition to the superconducting state versus applied magnetic field were derived and they were fitted using the formula: $\Delta T=C H^{m}+\Delta T_{0}$. The irreversibility fields as a function of temperature were obtained and fitted by the de Almeida and Thouless-like equation: $H_{\mathrm{irr}}=H_{\mathrm{irr} 0}\left(1-T / T_{c 0}\right)^{n}$. The irreversibility field at the liquid nitrogen temperature was calculated and it is $H_{\text {irr }}=43.8 \mathrm{kOe}$ when the applied magnetic field is parallel to the $c$-axis.
\end{abstract}

DOI: 10.12693/APhysPolA.131.1018

PACS/topics: 74.72.--h, 74.78.--w, 74.25.Op, 74.25.Qt, 74.78.Bz, 74.25.Qt

\section{Introduction}

The advanced development of the preparation of high temperature superconductors (HTS) films and tapes has opened new opportunities for large-scale applications including power system components such as electrical motors, generators, power transmission cables, transformers, magnets, and superconducting magnetic energy storage devices [1-3]. Extensive investigations have been focused on optimizing the fabrication and processing techniques of HTS wires and tapes [4] giving the high values of critical current densities and irreversibility fields. One of the highest reported critical current densities of the first generation (1G) bismuth based tapes was about $115 \mathrm{kA} \mathrm{cm}^{-2}$ and the irreversibility fields are $H_{\text {irr }}=72.8 \mathrm{kOe}$ and $H_{\text {irr }}=5.5 \mathrm{kOe}$ for the parallel and perpendicular direction respectively at $77 \mathrm{~K} \mathrm{[5-7].} \mathrm{The}$ critical current densities of the second generation $(2 \mathrm{G})$ $\mathrm{REBaCuO}$ 1:2:3 based tapes reach over $10^{6} \mathrm{~A} \mathrm{~cm}^{-2}$ [8].

In this paper the temperature dependences of the magnetoresistance of the $c$-axis orientation $\mathrm{YBa}_{2} \mathrm{Cu}_{3} \mathrm{O}_{\delta}$ thin film prepared on $\mathrm{MgO}$ substrate were studied and the field dependences of the transition width as well as temperature dependences of the irreversibility fields are reported and analyzed.

\section{Experiment}

The sample was prepared by the pulsed laser deposition (PLD) on single crystal (100) one side polished MgO

*corresponding author; e-mail: wmwoch@agh.edu.pl substrate. PLD setup (Neocera) was running on excimer laser (Coherent COMPexPro 110F) with a wavelength of $248 \mathrm{~nm}$. Pulse energy was $200 \mathrm{~mJ}$ and repetition rate was $10 \mathrm{~Hz}$. During deposition substrate temperature and $\mathrm{O}_{2}$ partial pressure were set to $750{ }^{\circ} \mathrm{C}$ and 100 mTorr, respectively. Deposition lasted 50 min. After deposition $\mathrm{O}_{2}$ partial pressure was set to 300 Torr and sample was cooled to $400{ }^{\circ} \mathrm{C}$ with ramp $3{ }^{\circ} \mathrm{C} / \mathrm{min}$ and then annealed in this temperature for $30 \mathrm{~min}$. The target used during film preparation was made from powder obtained by the standard solid-state reaction technique, pressed at a pressure of $0.7 \mathrm{GPa}$ and sintered at $940^{\circ} \mathrm{C}$ for $72 \mathrm{~h}$. The distance between target and substrate was $9 \mathrm{~cm}$. The film thickness was determined using X-ray fluorescence (XRF) spectrometer and it was $170 \mathrm{~nm}$. X-ray diffraction (XRD) on the film on $\mathrm{MgO}$ substrate showed excellent $c$-axis orientation and no secondary phases were detected in the XRD spectra [9].

The temperature dependences of the magnetoresistance were measured using the standard four-points ac method option of the Quantum Design PPMS apparatus with $90 \mathrm{kOe}$ superconducting magnet. The measurements were carried out for the $c$-axis parallel to the applied magnetic field.

\section{Results and discussion}

The temperature dependences of the magnetoresistance for the parallel magnetic field orientations with respect to the $c$-axis are shown in Fig. 1.

At the zero field the critical temperatures for this tape are as follows: $T_{c 50 \%}=87.5 \mathrm{~K}, T_{c 0}=85.8 \mathrm{~K}$ and the width of superconducting transition is $\Delta T_{0}=1.8 \mathrm{~K}$. One can notice a significant broadening of the superconducting transition with the application of the external 


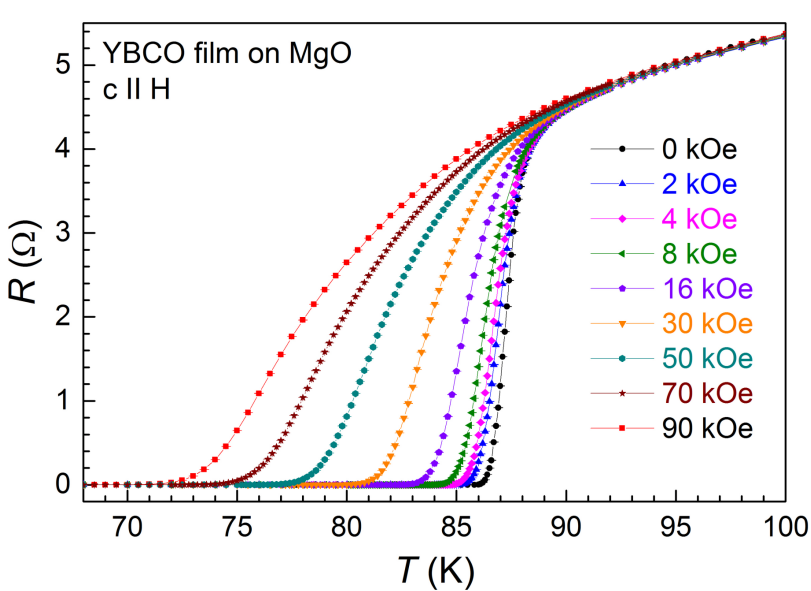

Fig. 1. Temperature dependences of the magnetoresistance of the $c$-axis orientation $\mathrm{YBa}_{2} \mathrm{Cu}_{3} \mathrm{O}_{\delta}$ thin film on $\mathrm{MgO}$ for the parallel magnetic field orientations with respect to the $c$-axis.

magnetic field, which is typical for HTS [10, 11]. Some authors try to explain this phenomenon with the vortex structure and flux motion mechanism $[12,13]$. One can expect that the resistive transition from the normal to the superconducting state of HTS hides many interesting physical phenomena [14] and this is possibly a key to understand the mechanism of a superconductivity of these materials. From this point of view the attention is paid to the width of the resistive transition that is usually defined as: $\Delta T=T_{90 \%}-T_{10 \%}$ and its dependence on the applied magnetic field. To take advantage of the experimental curves that are shown in Fig. 1 the widths of the transition versus applied magnetic fields were derived and they are shown in Fig. 2. One can notice that at the zero applied magnetic field the width of the resistive transition is small and rises remarkably when the magnetic field is increased. The width of the resistive transition starts from $\Delta T_{0}=1.8 \mathrm{~K}$ at the zero applied magnetic field and reaches $\Delta T=12.2 \mathrm{~K}$ at $90 \mathrm{kOe}$. The width of the resistive transition can generally be described in the following form [14]:

$$
\Delta T=C H^{m}+\Delta T_{0},
$$

where $m=2 / 3$ and $\Delta T_{0}$ means the width at the zero applied magnetic field. The exponent $m$ is related to the exponent $n$ that appears in the temperature dependence of the irreversibility field by the relation: $m=1 / n$. The coefficient $C$ depends on the critical current at zero magnetic field as well as on the critical temperature. The experimental data presented in Fig. 2 were well fitted using Eq. (1) (the solid lines in Fig. 2). For each direction the two parameters $m$ and $C$ were used as the fit parameters in Eq. (1). The width of the resistive transition at the zero applied magnetic field $\Delta T_{0}=1.8 \mathrm{~K}$ was taken from the experimental data. The parameters $m$ and $\mathrm{C}$ obtained from the fitting procedure are $0.84 \pm 0.03$ and $0.24 \pm 0.03$, respectively. The critical exponent derived from this fit is generally greater than the theoretical $m=2 / 3$ in Eq. (1). The greater parameter $m$ corresponds to a more three-dimensional vortex structure [15] and it means that the pinning force is not too strong. On the other hand, the density of the critical current of this film obtained from the ac susceptibility using the Bean model reach high value: $J_{c}=1.2 \times 10^{7} \mathrm{~A} / \mathrm{cm}^{2}$ at $77 \mathrm{~K}$ in the self magnetic field [16].

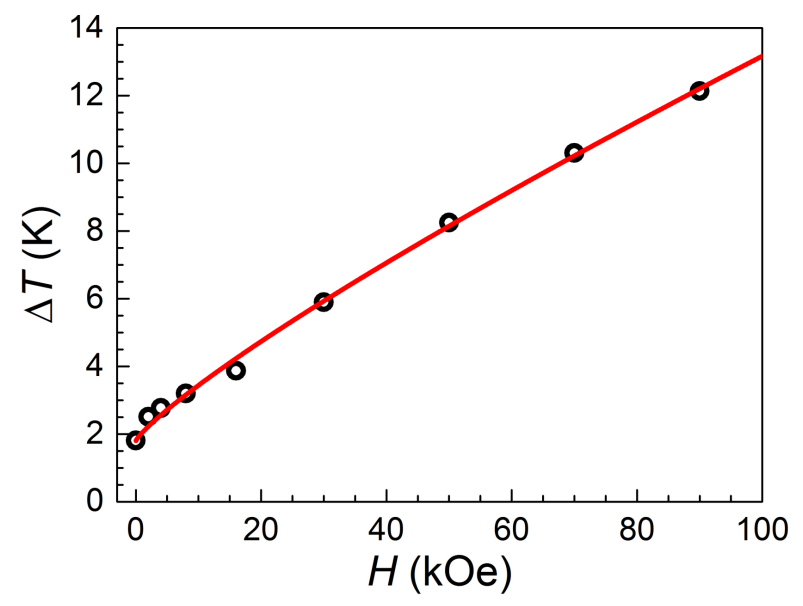

Fig. 2. Width of the transition versus the applied magnetic field. Solid line is the fit to Eq. (1).

The most important curve on the $H-T$ phase diagram of HTS is the irreversibility line $[17,18]$ that separates the vortex glassy from the vortex liquid state. This line is determined by the temperature dependence of the irreversibility fields above that the flowing current forces the vortices to move. It means that energy dissipation appears and supercurrent vanishes. From this point of view the irreversibility field of HTS plays a similar role to that of the upper critical field in classical superconductors. The temperature dependence of the irreversibility fields can be described by the following relation:

$$
H_{\text {irr }}=H_{\text {irr } 0}\left(1-\frac{T}{T_{c 0}}\right)^{n},
$$

where $H_{\text {irro }}$ is the irreversibility field at $0 \mathrm{~K}, T_{c 0}$ is the zero critical temperature at zero magnetic field and the exponent $n$, which is theoretically $3 / 2$, can vary in the wide range and depends on the vortex properties. One can assume [19] that the temperature at which the whole sample stayed superconducting (resistance equal to zero) at the given magnetic field separates the reversibility from the irreversibility region. The data $H_{\text {irr }}(T)$ were extracted from the magnetoresistance measurements and they are shown in Fig. 3.

The experimental data of $H_{\mathrm{irr}}(T)$ were successfully fitted using Eq. (2) with the two fit parameters: $n$ and $H_{\text {irro }}$ (solid lines in Fig. 3). The zero field critical temperature $T_{c 0}=85.8 \mathrm{~K}$ was taken from the experiment. The fit procedure delivers the following fit parameters values: $n=1.17 \pm 0.02$ and $H_{\text {irro }}=650 \pm 22 \mathrm{kOe}$. This value of the exponent in Eq. (2) is comparable to the inverse of the exponent $m$ defined in Eq. (1) that gives 1.19. Taking advantage of the fit parameters the irreversibility 


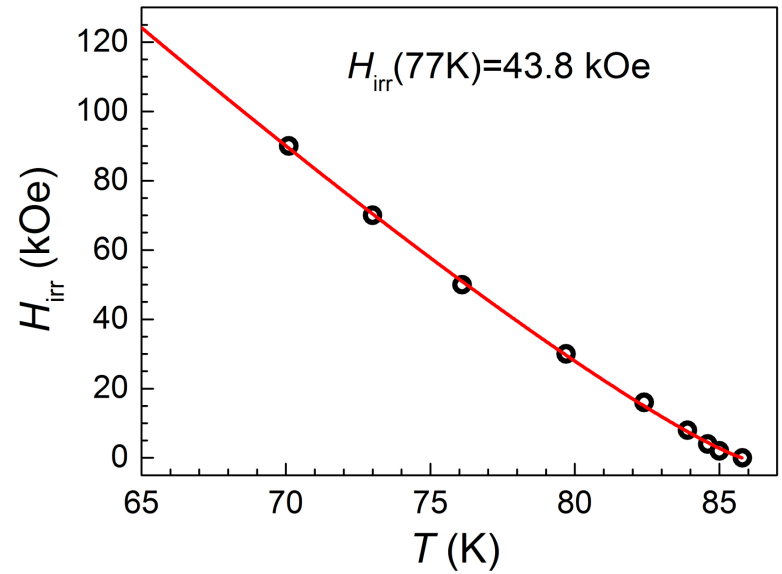

Fig. 3. Irreversibility fields as a function of temperature. Solid line is the fit to Eq. (2).

field at the liquid nitrogen temperature was calculated and it is $H_{\mathrm{irr}}=43.8 \mathrm{kOe}$.

\section{Conclusions}

The $c$-axis orientation $\mathrm{YBa}_{2} \mathrm{Cu}_{3} \mathrm{O}_{\delta}$ film on $\mathrm{MgO}$ possesses good superconducting parameters: the critical temperature $T_{c 50 \%}=87.5 \mathrm{~K}$, the width of the superconducting transition $\Delta T_{0}=1.8 \mathrm{~K}$ and the high critical current density $J_{c}=1.2 \times 10^{7} \mathrm{~A} / \mathrm{cm}^{2}$ at $77 \mathrm{~K}$ in the own magnetic field. The thickness of the film is $170 \mathrm{~nm}$. The width of superconducting transition versus magnetic field was fitted using Eq. (1). The parameters $m$ and $C$ obtained from the fitting procedure are $0.84 \pm 0.03$ and $0.24 \pm 0.03$, respectively. The critical exponent derived from this fit is generally greater than the theoretical $m=2 / 3$ and it corresponds to a more three-dimensional vortex structure. It means that the pinning force is not too strong. On the other hand, the density of the critical current of this film reaches really high value. The experimental data of $H_{\text {irr }}(T)$ were successfully fitted using Eq. (2) with the two fit parameters: $n$ and $H_{\text {irro. The }}$ fit procedure delivers the following fit parameters values: $n=1.17 \pm 0.02$ and $H_{\text {irro }}=650 \pm 22 \mathrm{kOe}$. The value of the exponent $n$ is comparable to the inverse of the exponent $m: 1 / m=1.19$. Taking advantage of the fit parameters the irreversibility field at $77 \mathrm{~K}$ was calculated and it is $H_{\mathrm{irr}}=43.8 \mathrm{kOe}$ when the applied magnetic field is parallel to the $c$-axis.

\section{Acknowledgments}

This work was supported by the Polish Ministry of Science and Higher Education and its grants for Scientific Research.

\section{References}

[1] H.J.M. der Brake, F. Buchholz, G. Burnell, T. Claeson, D. Crete, P. Febvre, G.J. Gerritsma, H. Hilgenkamp, R. Humphreys, Z. Ivanov, W. Jutzi, M.I. Khabipov, J. Mannhart, G-H. Meyer, J. Niemeyer, A. Ravex, H. Rogalla, M. Russo, J. Satchell, M. Siegel, M. Topfer, F.H. Uhlmann, JC. Villegier, E. Wikborg, D. Woinkler, A.B. Zorin, Physica C 439, 1 (2006).

[2] N. Ayai, M. Ueyama, T. Kato, S. Kobayashi, T. Mikumo, T. Kaneko, T. Hikata, K. Hayashi, H. Takei, in: Advances in Superconductivity XII, , Eds. T. Yamashita, K. Tanabe, Springer-Verlag, Tokyo 2000, p. 631.

[3] Y. Nagasaki, T. Nakamura, I. Funaki, Y. Ashida, H. Yamakawa, Physica C 492, 96 (2013).

[4] See for instance: J. Azman, H. Abdullah, R. AbdShukor, Adv. Condens. Matter Phys. 2014, 1 (2014) and references therein.

[5] M. Letovic, P. Krishnaraj, N.G. Eror, U. Balachandran, Physica C 242, 246 (1995).

[6] L. Masur, D. Parker, M. Tanner, E. Podtburg, D. Buczek, J. Scudiere, P. Caracino, S. Spreafico, P. Corsaro, M. Nassi, IEEE Trans. Appl. Supercond. 11, 3256 (2001).

[7] W.M. Woch, M. Chrobak, M. Kowalik, R. Zalecki, J. Przewoźnik, Cz. Kapusta, J. Supercond. Novel Magn. 29, 2333 (2016).

[8] See for instance: S. Yoon, J. Kim, H. Lee, S. Hahn, S.-H. Moon, Supercond. Sci. Technol. 29, 337 (2016).

[9] M. Chrobak, Ph.D. Thesis, AGH UST, Kraków 2015, p. 71.

[10] W.M. Woch, R. Zalecki, A. Kołodziejczyk, H. Sudra, G. Gritzner, Supercond. Sci. Technol. 21, 085002 (2008).

[11] W.M. Woch, M. Chrobak, R. Zalecki, A. Kołodziejczyk, Acta Phys. Pol. A 126, 328 (2014).

[12] T.T.M. Palstra, B. Batlogg, L.F. Schneemeyer, J.V. Waszczak, Phys. Rev. Lett. 61, 1662 (1988).

[13] W.K. Kwok, U. Welp, G.W. Crabtree, K.G. Vandervoort, R. Hulscher, J.Z. Liu, Phys. Rev. Lett. 64, 966 (1990).

[14] W.M. Woch, R. Zalecki, M. Chrobak, A. Kołodziejczyk, G. Gritzner, Acta Phys. Pol. A 121, 789 (2012).

[15] Y. Yeshurun, A.P. Malozemoff, Phys. Rev. Lett. 60 , 2202 (1988)

[16] W.M. Woch, R. Zalecki, M. Chrobak, A. Kołodziejczyk, Acta Phys. Pol. A 127, 315 (2015).

[17] K.A. Müller, M. Takashige, J. Bednorz, Phys. Rev. Lett. 58, 1143 (1987).

[18] J.R.L. de Almeida, D.J. Thouless, J. Phys. A 11, 983 (1978).

[19] W.M. Woch, R. Zalecki, A. Kołodziejczyk, O. Heiml, G. Gritzner, Physica C 434, 17 (2006). 